Pacific Journal of Mathematics

INJECTIVE HULLS OF SEMI-SIMPLE MODULES OVER 


\title{
INJECTIVE HULLS OF SEMI-SIMPLE MODULES OVER REGULAR RINGS
}

\author{
A. K. TIWARY
}

The object of this paper is to provide an explicit construction of the injective hull of a semi-simple module over a commutative regular ring.

The existence of injective hulls of an arbitrary module $M$ and their uniqueness upto isomorphism over $M$ was shown by B. Eckmann and A. Schopf in 1953 [6]. But only in few cases these hulls have been described explicitly $[1,2]$.

In the special case when the ring is regular as well as Noetherian, the problem is already solved since over such a ring every module is known to be semi-simple [9] and hence is its own injective hull [11, 10]. To begin with we show that every monotypic component of the module is injective and then prove a topological lemma about $T_{1}$-spaces. The Zariski topology of the maximal ideal space of the basic ring being $T_{1}$, we make use of the lemma to obtain the desired construction of an injective hull of the module. We show by an example that a semisimple module over a regular ring need not always be injective and obtain finally a necessary and sufficient condition for the injectivity of the module.

Definition 1. A ring $R$ is called (von Neumann) regular if for every $a \in R$, there exists an element $x \in R$ such that $a x a=a$. This condition reduces to $a^{2} x=a$ if $R$ is commutative. A Boolean ring is an example of a commutative regular ring. It is well known that a commutative ring $R$ with unit is regular if and only if every simple $R$-module is injective [11].

Throughout this paper we shall consider $R$ to be a commutative regular ring with unit 1 . Let $\Omega$ denote the set of maximal ideals of $R$. For each $a \in R$ define $\Omega_{a}$ by $\Omega_{a}=\{P \in \Omega \mid a \notin P\}$. It follows that $\Omega_{a} \cap \Omega_{b}=\Omega_{a b}$. Thus $\Omega$ can be made into a topological space with $\left\{\Omega_{a} \mid a \in R\right\}$ as the system of basic open sets. This topology of $\Omega$ is known as the Zariski topology. $\Omega$ is clearly a $T_{1}$-space since if $P$ and $Q$ are any two distinct points in $\Omega$, there exists $a \in P-Q$ which implies that $\Omega_{a}$ is a neighbourhood of $Q$ not containing $P$.

Definition 2. Let $M$ be a semi-simple $R$-module. For any simple submodule $S$ of $M$, there exists exactly one $P \in \Omega$ with $S \cong R / P$. The 
sum of all those simple submodules of $M$ which are isomorphic to $R / P$, will be denoted by $M_{P}$ and will be called the $R / P$-monotypic componet of $M$. The support of $M$, to be denoted by Supp $(M)$ is the set of all those maximal ideals $P$ in $\Omega$ for which $M_{P}$ is nonzero.

In our discussion $M$ will always denote a semi-simple $R$-module with supp $(M)=S$. As usual for any function $f$, the symbol supp $(f)$ will mean the set of all those elements in domain $(f)$ for which $f(x) \neq 0$. We shall write $E=H(M)$ to express the fact that $E$ is an injective hull of $M$. Where no ambiguity can arise, we let $H(M)$ stand for an arbitrary injective hull of $M$. If $\alpha$ is any cardinal number and $L$ any module, the sombol $\alpha \odot L$ will stand for the external sum of $\alpha$ copies of $L$.

THEOREM 1. For any $P \in S$, the associated monotypic component $M_{P}$ is an injective module.

Proof. Let $\alpha$ be the length of $M_{P}$ and $T$ a set with $|T|=\alpha$. Then $M_{P} \cong \alpha \odot R / P=E$. Let $\pi$ be the set of all functions from $T$ into $R / P$. Now each factor $R / P$ of $\pi$ being injective [11], $\pi$ is injective; hence there exists an $H(E) \subseteq \pi$. Without loss of generality we can take $\alpha$ to be an infinite cardinal. Assume $E$ is not injective. Then $E \subset H(E) \leqq \pi$. Take any element $f \in H(E)-E$. Since $H(E)$ is an essential extension of $E$, one has $R f \cap E \neq 0$ which implies $0 \neq r f \in E$ for some $r \in R-P$. As $R / P$ is a field and $f(t) \neq 0$ for infinitely many $t \in T$, we have $0 \neq(r+P) f(t)=r f(t)$ for infinitely many $t \in T$. But this contradicts the fact that $r f \in E$. Hence $E$ is injective.

REMARK 1. $\prod_{P \in S} M_{p}$ is injective since each factor $M_{P}$ is injective.

Definition 3. Let $X$ be any topological space and $A$ any subset of $X$. An element $x \in A$ is called an isolated point of $A$ if there exists a neighbourhood $U$ of $x$ such that $U \cap A=\{x\}$, i.e., if $\{x\}$ is an open set in the relative topology of $A$. A subset $A$ of $X$ is said to be discrete if every element $x$ in $A$ is an isolated point of $A$.

LeMma 1. Let $f \in \prod_{P \in S} M_{P}$ and $a \in R$ such that $0 \neq a f \in \bigoplus_{P \in S} M_{P}$, then every element in supp $(a f)$ is an isolated point of supp $(f)$.

Proof. Let $\operatorname{supp}(a f)=\left\{P_{1}, P_{2}, \cdots, P_{n}\right\}$ where $P_{i} \neq P_{j}$ if $i \neq j$. This implies that there exist elements $a_{i} \in P_{i}-P_{1}(i=2,3, \cdots, n)$. Put $b=a a_{2} a_{3} \cdots a_{n}$. Then $b \notin P_{1}$ and $b \in P$ for each $P \in \operatorname{supp}(f)$ with $P \neq P_{1}$. Hence $\Omega_{b} \cap \operatorname{supp}(f)=\left\{P_{1}\right\}$ showing that $P_{1}$ is an isolated 
point of $\operatorname{supp}(f)$. Similar argument will prove that $P_{2}, \cdots, P_{n}$ are also isolated points of $\operatorname{supp}(f)$.

REMARK 2. It follows from the lemma that the support of any nonzero element in an essential extension of $\bigoplus_{P \in S} M_{P}$ contains an isolated point.

Lemma 2. Let $E$ be a proper essential extension of $\bigoplus_{P \in S} M_{P}$. Then for any $f \in E-\bigoplus_{P \in S} M_{P}$, supp $(f)$ contains infinitely many isolated points.

Proof. Since $E$ is an essential extension of $\bigoplus_{P \in S} M_{P}$ and $0 \neq f \in E$, we can find an element $a \in R$ such that $0 \neq a f \in \bigoplus_{P \in S} M_{P}$. Let supp $(a f)=\left\{P_{1}, P_{2}, \cdots, P_{n}\right\}$. By Lemma 1 , each $P_{1}$ is an isolated point of $\operatorname{supp}(f)$. Choose an element $Q \in \operatorname{supp}(f)-\operatorname{supp}(a f)$. As $P_{i} \nsubseteq Q$, there exist elements $r_{i} \in P_{i}-Q(i=1,2, \cdots, n)$. Then

$$
r=r_{1} r_{2} \cdots r_{n} \in\left(P_{1} \cap P_{2} \cap \cdots \cap P_{n}\right)-Q \text {. }
$$

It follows that $0 \neq r f \in E$. Since for some $s \in R, 0 \neq s r f \in \bigoplus_{P \in S} M_{P}$, we can apply Lemma 1 to show that the elements in supp (srf) are isolated points of supp $(f)$ and they are all distinct from $P_{1}, P_{2}, \ldots, P_{n}$. Now supp $(f)$ being infinite, we can find an element in

$$
\operatorname{supp}(f)-(\operatorname{supp}(a f) \cup \operatorname{supp}(s r f))
$$

which will give rise to another set of finitely many elements isolated points of $\operatorname{supp}(f)$ each being different from the ones obtained before. Proceeding thus we get infinitely many isolated points of supp $(f)$. This proves the lemma.

We now prove the following topological fact about $T_{1}$-spaces:

Lemma 3. In any $T_{1}$-space $X$, if $A$ and $B$ are nonvoid subsets such that $A$ as well as every nonvoid subset of $B$ has an isolated point, then there exists an isolated point in $A \cup B$.

Proof. Let the complement of a subset $C$ of $X$ be denoted by $C^{\prime}$. Since $A$ is given to have an isolated point $p$, there exists an open neighbourhood $U$ of $p$ such that $U \cap A=\{p\}$. From

$$
U \cap\left(A \cup\left(B \cap U^{\prime}\right)\right)=U \cap A
$$

we conclude that $p$ is also an isolated point of $A \cup\left(B \cap U^{\prime}\right)$. If $B \cap U$ is empty, then $p$ is an isolated point of $A \cup B$ and so the lemma holds. We have therefore to consider only the case when $B \cap U$ is nonvoid. 
By hypothesis $B \cap U$ contains an isolated point $q$ which can be assumed to be distinct from $p$ without any loss in generality. This assumption, together with the fact that $X$ is $T_{1}$ implies that $\{p\}^{\prime}$ is an open set containing $q$. Now $q$ being an isolated point of $B \cap U$, we have $V \cap B \cap U=\{q\}$ for some neighbourhood $V$ of $q$. Thus we obtain

$$
U \cap V \cap\{p\}^{\prime} \cap(A \cup B)=U \cap V \cap\{p\}^{\prime} \cap B=\{q\} \cap\{p\}^{\prime}=\{q\} .
$$

Since $U \cap V \cap\{p\}^{\prime}$ is a neighbourhood of $q$, the above relation implies that $q$ is an isolated point of $A \cup B$.

REMARK 3. From Lemma 3 we immediately have the following

(i) Let $B$ be a discrete subset of a $T_{1}$-space $X$ and $A$ any subset of $X$ with an isolated point, then $A \cup B$ has an isolated point.

(ii) If $A$ and $B$ are nonvoid subsets of a $T_{1}$-space $X$ with the property that each of their nonvoid subsets has an isolated point then $A \cup B$ has the same property.

Lemma 4. Let $A=\bigcup_{i \in I} A_{i}$ where each $A_{i}$ is without an isolated point. Then $A$ has no isolated point.

Proof. Suppose $A$ has an isolated point $p$. Then $p \in A_{i}$ for some $i \in I$ and $\{p\}=U \cap A$ for some neighbourhood $U$ of $p$. Hence $\{p\}=$ $U \cap A_{i}$ contrary to the hypothesis that $A_{i}$ is without an isolated point. Thus $A$ has no isolated point.

Lemma 5. If $A$ has no isolated point, then $\bar{A}$, the closure of $A$ also has no isolated point.

Proof. Assume $p$ is an isolated point in $\bar{A}$ with $V \cap \bar{A}=\{p\}$ for some neighbourhood $V$ of $p$, then $p \in \bar{A} \cap A^{\prime}$ implies the existence of an element $q \in V \cap A \subseteq V \cap \bar{A}$ with $q$ distinct from $p$, a contradiction. Hence $A$ has no isolated point.

REMARK 4. We know that the semi-simple module $M=\sum_{P \in S} M_{P}$ (direct) hence $M \cong \bigoplus_{P \in S} M_{P}$. Since the injective module $\Pi_{P \in S} M_{P}$ contains $\bigoplus_{P \in S} M_{P}$ as a submodule, it also contains an $H\left(\bigoplus_{P \in S} M_{P}\right)$. Thus to find an injective hull of $M$, it is sufficient to obtain one of $\bigoplus_{P \in S} M_{P}$ inside $\prod_{P \in S} M_{P}$. This is done in the following:

THEOREM 2. Let $H=\left\{f \in \prod_{P \in S} M_{P} \mid\right.$ Every nonvoid subset of supp $(f)$ has an isolated point $\}$. Then $H$ is an injective hull of $\bigoplus_{P \in S} M_{P}$.

Proof. Let $f, g$ be any two elements in $H$, then since 
$\operatorname{supp}(f+g) \subseteq \operatorname{Supp}(f) \cup \operatorname{supp}(g)$, we have $f+g \in H$ by Remark 3 (ii) following Lemma 3. Now if a $\in R, f \in H$, then $\operatorname{supp}(a f)=\Omega_{a} \cap \operatorname{supp}(f)$ implies that $a f \in H$. Hence $H$ is an $R$-submodule of $\prod_{P \in S} M_{P}$ and it contains $\bigoplus_{P \in S} M_{P}$ since every nonvoid subset of a finite set is discrete. Now let $0 \neq f \in H$, then $\operatorname{supp}(f)$ is nonempty and hence contains an isolated point $P$ so that for some

$$
a \in R, \operatorname{supp}(a f)=\Omega_{a} \cap \operatorname{supp}(f)=\{p\} .
$$

Thus $0 \neq a f \in \bigoplus_{P \in S} M_{P}$. Hence $H$ is an essential extension of $\bigoplus_{P \in S} M_{P}$.

As to the injectivity of $H$ assume by way of contradiction that $H$ has a proper essential extension $E$. Then $H \subset E \cong \prod_{P \in S} M_{P}$. Take $f \in E, f \in H$. Then there exists a nonvoid subset of $\operatorname{supp}(f)$ without isolated points. Denote by $X$, the union of all those subsets of $\operatorname{supp}(f)$ which have no isolated points. By Lemma 4, $X$ has no isolated point. Let $Y=\operatorname{supp}(f) \cap X^{\prime}$ where $X^{\prime}$ is the complement of $X$ in $S$. Then $Y$ is nonvoid since by Remark 2 , Lemma $1, \operatorname{supp}(f)$ contains an isolated point which cannot belong to $X$. Thus $\operatorname{supp}(f)=$ $X \cup Y$ is a decomposition of $\operatorname{supp}(f)$ into disjoint nonempty subsets $X$ and $Y$. Moreover every nonvoid subset of $Y$ contains an isolated point for otherwise it will have to be contained in $X$ which is not possible. Now for any subset $A \cong \operatorname{supp}(f)$, define $f_{A}$ to be the function such that

$$
f_{A}(P)=\left\{\begin{array}{cl}
f(P) & \text { if } P \in A \\
0 & \text { if } P \in S-A
\end{array}\right.
$$

we can then write $f=F_{X}+f_{Y^{\prime}}$. Since $\operatorname{supp}\left(f_{Y^{\prime}}\right)=Y$, one has $f_{Y^{*}} \in H$ and hence from $f_{X}=f-f_{Y}$, it follows that $f_{X} \in E$. The fact that $f_{X}$ is a nonzero element in an essential extension $E$ of $\bigoplus_{P \in S} M_{P}$, then implies that $X=\operatorname{supp}\left(f_{X}\right)$ has an isolated point. We thus arrive at a contradiction. Hence $H$ is injective. This completes the proof.

CoRollary 1. $\prod_{P \in S} M_{P}$ is an injective hull of $\bigoplus_{P \in S} M_{P}$ if and only if every nonvoid subset of $S$ has an isolated point. In particular if $S$ is discrete in $\Omega$, then $\prod_{P \in S} M_{P} \cong H(M)$.

Proof. If $S$ has the property that each of its nonvoid subsets has an isolated point, then for every $f \in \prod_{P \in S} M_{P}$, supp $(f)$ has the same property. Hence by Theorem $2, \Pi_{P \in S} M_{P}=H\left(\bigoplus_{P \in S} M_{P}\right)$. On the other hand let $\prod_{P \in S} M_{P}=H\left(\bigoplus_{P \in S} M_{P}\right)$. Suppose that some non-empty subset $A$ of $S$ has no isolated point. Then $A$ must be an infinite set. We can find a function $f \in \prod_{P \in S} M_{P}$ with $\operatorname{supp}(f)=A$. Then $f \in \bigoplus_{P \in S} M_{P}$ and hence $f \neq 0$. Since $\prod_{P \in S} M_{P}$ is an essential extension of $\bigoplus_{P \in S} M_{P}$, by 
Remark 2, supp $(f)$ has an isolated point contrary to the assumption that $A$ has no isolated point. Hence every nonvoid subset of $S$ has an isolated point. The last part of the corollary follows immediately from the fact that every element in a discrete set is an isolated point.

Corollary 2. If $S$ contains only principal ideals, then

$$
\prod_{P \in S} M_{P}=H\left(\bigoplus_{P \in S} M_{P}\right) \text {. }
$$

Proof. Let $R a$ be any maximal ideal in $S$. If $P$ in $S$ is different from $R a$, then $a \notin P$ since $a \in P$ would mean $R a \subseteq P$, hence $R a=P$, a contradiction. Regularity of $R$ implies that $a=a^{2} x$ for some $x \in R$. Since $0=a(1-a x)$ belongs to every $P$ in $S, 1-a x$ belongs to every element in $S$ different from $R a$. Also $1-a x \notin R a$ since other wise $1 \in R a$. It follows that $\Omega_{1-a x} \cap S=\{R a\}$. Thus every element in $S$ is an isolated point. By Corollary 1 , we have $\prod_{P \in S} M_{P}=$ $H\left(\oplus_{P \in S} M_{P}\right)$.

REMARK 5. For any module $M$ over a regular and Noetherian ring $R, \Pi_{P \in S} M_{P}=H\left(\bigoplus_{P \in S} M_{P}\right)=\bigoplus_{P \in S} M_{P}$ since every ideal of $R$ is a principal ideal [9] and every $R$-module is injective [10, 11].

COROLlary 3. There exist semi-simple modules over a regular ring which are not injective.

Proof. Let $R_{0}$ be the two-element Boolean ring $\left\{0, e_{0}\right\}, I$ an infinite index set and $R$, the set of all functions $f: I \rightarrow R_{0}$. Then $R$ is a complete Boolean ring and hence a commutative regular ring. For each $\alpha \in I$, define $P_{\alpha}$ by $P_{\alpha}=\{f \in R \mid f(\alpha)=0\}$. It is easily seen that $P_{\alpha}$ is a maximal ideal of $R[7]$. Let $M=\bigoplus_{\alpha \in I} R / P_{\alpha}$. Then $M$ is a semi-simple module with $\operatorname{Supp}(M)=\left\{P_{\alpha} \mid \alpha \in I\right\}$. Take any $P_{\alpha_{0}} \in \operatorname{Supp}(M)$ and define $f$ by

$$
f(\alpha)=\left\{\begin{array}{lll}
e_{0} & \text { if } & \alpha=\alpha_{0} \\
0 & \text { if } & \alpha \neq \alpha_{0}
\end{array}\right.
$$

then $f \in R-P_{\alpha_{0}}$ and $f \in P_{\beta}$ for all $\beta \in I$ with $\beta \neq \alpha_{0}$. Thus

$$
\Omega_{f} \cap \operatorname{Supp}(M)=\left\{P_{\alpha_{0}}\right\}
$$

which implies that $\operatorname{Supp}(M)$ is discrete. Hence by Corollary 1, $\prod_{\alpha \in I}\left(R / P_{\alpha}\right)=H\left(\bigoplus_{\alpha \in I}\left(R / P_{\alpha}\right)\right.$. The fact that $I$ is infinite then shows that $\bigoplus_{\alpha \in I}\left(R / P_{\alpha}\right)$ is not injective.

Corollary 4. If $S=A \cup D_{1} \cup D_{2} \cup \cdots \cup D_{n}$ where $A$ has an 
isolated point and $D_{i}(i=1,2, \cdots, n)$ are discrete sets, then $\prod_{P \in S} M_{P} \cong$ $H(M)$.

Proof. It follows immediately from Lemma 3 and Corollary 1.

In Corollary 3 we have a concrete example showing that not every semi-simple $R$-module is injective. It is therefore worthwhile to ask under what conditions a semi-simple $R$-module is injective. The following theorem gives a characterisation for the injectivity of a semisimple module.

THEOREM 3. $M$ is injective if and only if $S$ has only finite discrete subsets.

Proof. Let $M$ be injective. Assume that $D \subseteq S$ is an infinite discrete subset. We can find $f \in \Pi_{P \in S} M_{P}$ with $\operatorname{supp}(f)=D$. Since $D$ is infinite, $f \notin \bigoplus_{P \in S} M_{P}$. The fact that $\operatorname{supp}(f)$ is discrete implies by Theorem 2, that $f \in H\left(\bigoplus_{P \in S} M_{P}\right)=\bigoplus_{P \in S} M_{P}$ and so we get a contradiction. Hence $S$ contains only finite discrete subsets.

Conversely suppose that $S$ has only finite discrete subsets. Assume that $M$ is not injective. Then $\bigoplus_{P \in S} M_{P}$ has a proper essential extension $E$ inside $\Pi_{P \in S} M_{P}$. Hence for any $f \in E-\bigoplus_{P \in S} M_{P}$, supp $(f)$ contains an infinite discrete subset by Lemma 2. This contradiction then proves that $M$ is injective.

Added in Proof.

REMARK 6. Under the assumptions of Theorem $3, S$ is a compact subset of $\Omega$.

Proof. Let $S \leqq U_{i \in I} \Omega_{a_{i}}$ so that $S=U_{i \in I}\left(S \cap \Omega_{a_{i}}\right)$ where we assume without loss of generality that each $S \cap \Omega_{a_{i}}$ is nonvoid. For each $i$ in $I$, pick one $P_{i}$ from $S \cap \Omega_{a_{i}}$ and let $A$ be the set of all such $P_{i}$. Then $\Omega_{a_{i}} \cap A=\left\{P_{i}\right\}$ for each $i$ in $I$. This implies that $A$ is a discrete subset of $S$ and hence by Theorem 3, $A$ is finite. Consequently $S$ is compact.

As a consequence of the above remark, we obtain as a corollary of Theorem 3, the following result of J. Levine, announced in an abstract in the Notices:

COROLlary. (Levine) If an injective module $M$ over a commutative regular ring $R$ is a direct sum of simple submodules, then there are only finitely many nonisomorphic simples in the sum.

Proof. Let $M^{*}=\sum_{P} X_{P}$ be the sum of nonisomorphic simple submodules in the direct sum decomposition of $M$. Then for each $X_{P}$, 
there exists exactly one $P$ in $S$ with $X_{P}$ isomorphic to $R / P$ and hence the $R / P$-monotypic component of $M^{*}$ is $X_{P}$. Moreover, $M^{*}$ being a direct summand of $M$, is injective and, therefore, by Remark 6, its support $S^{*}$ is compact. Any nonvoid subset of $S^{*}$ also has this property since it is injective. We propose to show that $S^{*}$ is discrete. Take any $P$ in $S^{*}$ and let $\{P\}^{\prime}$ be the complement of $\{P\}$ in $S^{*}$. Then $\{P\}^{\prime}$ being open and compact, we have $\{P\}^{\prime}=U_{i \in 1}^{n} S_{c_{i}}$, where $S_{c_{i}}=$ $\Omega_{c_{i}} \cap S^{*}$. Now, $c_{i}$ in $R$ implies that there exists $x_{i}$ in $R$ with $c_{i}=c_{i}^{2} x_{i}$ $i=1,2, \cdots, n$. Put $d_{i}=1-c_{i} x_{i}$. Then from $c_{i} d_{i}=0$, it follows that $d=d_{1} d_{2} \cdots d_{n}$ belongs to every $Q$ in $S^{*}$, different from $P$ and does not belong to $P$. Hence $\{P\}=S_{d}$. Thus every point in $S^{*}$ is an isolated point as was required. By Theorem 3 we have $S^{*}$ finite.

Remark 7. Theorem 1 is a special case of a more general Proposition of C. Faith [Proposition 3, Rings with ascending condition on annihilators, Nagoya Math. J. 27 (1966), 179-181]. Let a module $M$ be called $\Sigma$-injective if it is injective and every direct sum of copies of $M$ is also injective. Then Proposition 3 of Faith has the following corollaries:

CoROllaRY 1. Let $R$ be any ring, and let $M$ be any injective simple module. Then if $M$ is finite dimensional over the field $K=$ End $M_{R}$, then $M$ is $\Sigma$-injective.

CoROllary 2. If $R$ is any commutative ring, and $M$ is an injective simple module, then $M$ is $\Sigma$-injective.

Theorem 1 is a special case of Corollary 2 when $R$ is a regular ring.

REMARK 8. Corollary 3 of Theorem 2 provides an example of a semisimple module over a commutative regular ring which is not injective. C. Faith has sketched an example of a simple module over a noncommutative regular ring which is not injective [Chapter 15, "Lectures on Injective Modules and Quotient Rings" Springer Verlag, New York 1967].

I should like to express my grateful thanks to the referee for suggesting the addition of Remarks 6,7 and 8 in proof.

This paper is a part of a doctoral dissertation submitted to McMaster University in 1966. I should like to express my indebtedness and grateful thanks to Professor B. Banaschewski under whose guidance this work was done. 


\section{REFERENCES}

1. B. Banaschewski, On the injective hulls of cyclic modules over Dedekind domains, Canad. Math. Bull. 9 (1966), 183-186.

2. - On coverings of modules, Math. Nachr. 31 (1966), 57-71.

3. N. Bourbaki, Algebra, Chap II, Herman, Paris, 1962.

4. H. Cartan and S. Eilenberg, Homological algebra, Princeton, 1956.

5. C. Chevalley, Fundamental concepts of algebra, Academic Press, 1956.

6. B. Eckmann and A. Schopf, Über injektive Moduln, Archiv der Math. 4 (1953), $75-78$.

7. L. Gillman and M. Jerison, Rings of continuous functions, Van Nostrand Company 1960 .

8. J. L. Kelley, General topology, Van Nostrand Company, 1955.

9. J. Lambek, Lectures on rings and modules, Blaisdell Publishing Company, 1966.

10. E. Matlis, Injective modules over Noetherian rings, Pacific. J. Math. 8 (1958), $511-528$.

11. A. Rosenberg and D. Zelinski, Finiteness of the injective hull, Math. Z. 70 (1959), 373-380.

Received November 28, 1968.

Banaras Hindu University 



\section{PACIFIC JOURNAL OF MATHEMATICS}

\section{EDITORS}

H. ROYDEN

Stanford University

Stanford, California

Richard Pierce

University of Washington

Seattle, Washington 98105
J. DUGUNDJI

Department of Mathematics

University of Southern California

Los Angeles, California 90007

BASIL GORDON

University of California

Los Angeles, California 90024

\section{ASSOCIATE EDITORS}
E. F. BECKENBACH
B. H. NEUMANN
F. WOLF
K. YosHIDA

\section{SUPPORTING INSTITUTIONS}

UNIVERSITY OF BRITISH COLUMBIA

CALIFORNIA INSTITUTE OF TECHNOLOGY

UNIVERSITY OF CALIFORNIA

MONTANA STATE UNIVERSITY

UNIVERSITY OF NEVADA

NEW MEXICO STATE UNIVERSITY

OREGON STATE UNIVERSITY

UNIVERSITY OF OREGON

OSAKA UNIVERSITY

UNIVERSITY OF SOUTHERN CALIFORNIA
STANFORD UNIVERSITY

UNIVERSITY OF TOKYO

UNIVERSITY OF UTAH

WASHINGTON STATE UNIVERSITY

UNIVERSITY OF WASHINGTON

*

AMERICAN MATHEMATICAL SOCIETY CHEVRON RESEARCH CORPORATION TRW SYSTEMS

NAVAL WEAPONS CENTER 


\section{Pacific Journal of Mathematics}

\section{Vol. 31, No. $1 \quad$ November, 1969}

James Burton Ax, Injective endomorphisms of varieties and schemes........

Richard Hindman Bouldin, A generalization of the Weinstein-Aronszajn

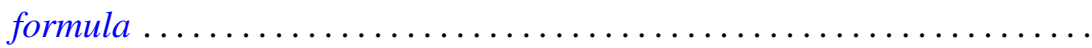

John Martin Chadam, The asymptotic behavior of the Klein-Gordon equation with external potential. II ...............................

Rina Hadass, On the zeros of the solutions of the differential equation

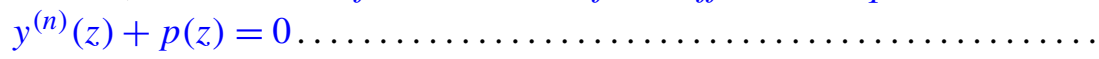

John Sollion Hsia, Integral equivalence of vectors over local modular lattices. II .............................................

Robert Hughes, Boundary behavior of random valued heat polynomial

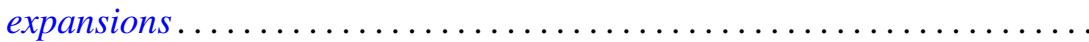

Surender Kumar Jain, Saad H. Mohamed and Surjeet Singh, Rings in which every right ideal is quasi-injective .........................

T. Kawata, On the inversion formula for the characteristic function .........

Erwin Kleinfeld, On right alternative rings without proper right ideals......

Robert Leroy Kruse and David Thomas Price, On the subring structure of

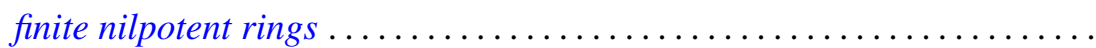

Marvin David Marcus and Stephen J. Pierce, Symmetric positive definite multilinear functionals with a given automorphism. .

William Schumacher Massey, Pontryagin squares in the Thom space of a bundle...

William Schumacher Massey, Proof of a conjecture of Whitney ...

John William Neuberger, Existence of a spectrum for nonlinear transformations

Stephen E. Newman, Measure algebras on idempotent semigroups ...

$\mathrm{K}$. Chandrasekhara Rao, Matrix transformations of some sequence spaces

Robert Bruce Schneider, Some theorems in Fourier analysis on symmetric

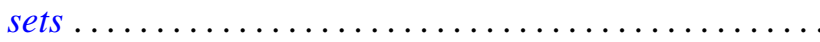

Ulrich F. K. Schoenwaelder, Centralizers of abelian, normal subgroups of hypercyclic groups...

Jerrold Norman Siegel, $G$-spaces, $H$-spaces and $W$-spaces

Robert Irving Soare, Cohesive sets and recursively enumerable Dedekind cuts...

Kwok-Wai Tam, Isometries of certain function spaces .... . .

Awadhesh Kumar Tiwary, Injective hulls of semi-simple modules over regular rings ....

Eldon Jon Vought, Concerning continua not separated by any nonaposyndetic subcontinuum .................... 